\title{
Medicación potencialmente inadecuada como factor de riesgo para el delirium en adultos mayores, en un servicio de emergencia
}

\author{
Potentially inappropriate \\ medication as a risk factor \\ for delirium in elderly, in an \\ emergency service
}

\author{
José Percy Amado-Tineo, ${ }^{1}$ \\ Rolando Vásquez-Alva, ${ }^{1}$ \\ Erwin Kraenau-Espinal, \\ Feodoro Julio Oscanoa-Espinoza ${ }^{3}$ \\ 1 Médico Internista, magíster en Docencia e Investigación en Salud. \\ Hospital Rebagliati. Lima, Perú. \\ 2 Doctor en Estadística. Universidad Nacional Mayor de San Marcos. \\ Lima, Perú. \\ 3 Médico Internista, doctor en Medicina. Hospital Almenara. Lima, Perú.
}

\section{RESUMEN}

Oвлеtivo. Evaluar la asociación del delírium (síndrome confusional agudo) con la medicación potencialmente inadecuada en adultos mayores. PACIENTES Y Método. Estudio prospectivo analítico de casos y controles. Muestreo aleatorio sistemático de pacientes adultos mayores (65 años o más) con delírium. Participantes: 102 casos y 136 controles; internados en el servicio de emergencia para adultos del Hospital Nacional Edgardo Rebagliati Martins, en Lima. Entrevista y evaluación al paciente o cuidador al ingreso. Instrumentos: CAM-ICU y STOPP. RESULTADOs. Se prescribieron 731 fármacos en 238 pacientes; en promedio, tres fármacos por paciente (combinación más frecuente: enalapril, ácido acetilsalicílico y furosemida).Se encontró medicación potencialmente inadecuada en $24,6 \%$ (30\% de casos y $20 \%$ de controles; $p=0,14)$, con mayor frecuencia glibenclamida, digoxina (más de $125 \mu \mathrm{g} / \mathrm{d}$ ), nifedipino de acción corta y diazepam. Los factores que tuvieron una asociación significativa $(p<0,05)$ con el delírium fueron edad mayor de 85 años, instrucción secundaria o menor, vivir solo, institucionalizado, internado durante el año previo, pluripatología, antecedente de enfermedad cerebrovascular, demencia, dependencia funcional, deshidratación moderada a grave $e$ infección al momento del ingreso. Se obtuvo odds ratio $>$ 1,0 para instrucción secundaria o menor, antecedente de enfermedad cerebrovascular, cirrosis hepática, demencia y deshidratación moderada a severa al ingreso. Conclusiones. Son factores de riesgo para el delírium la instrucción secundaria o menor, antecedente de enfermedad cerebrovascular, cirrosis hepática, demencia y deshidratación moderada-severa. La medicación potencialmente inadecuada no fue un factor de riesgo para el delírium en adultos mayores.

Palabras clave: prescripción inadecuada; delírium; síndrome confusional agudo; adulto mayor

\begin{abstract}
Oвлестіve. To evaluate the association of delirium (acute confusional state) with potentially inappropriate medication in older adults. Patients and Method. Analytical study of cases and controls. Systematic random sampling. Case: elderly patients with delirium. Participants: 102 cases and 136 controls; patients 65 years or older, admitted to the emergency service for adults Edgardo Rebagliati Martins National Hospital, in Lima. Interview and evaluate the patient or caregiver to income. Instruments: CAM-ICU and STOPP. RESULTs. 731 drugs were prescribed in 238 patients; three drugs per patient, on average; the most common: enalapril, furosemide and acetylsalicylic acid. Potentially inappropriate medication in $24.6 \%$ (30\% of cases and $20 \%$ of controls; $p=0.14$ ) were found. They were more often: glibenclamide, digoxin (125 $\mu \mathrm{g} / \mathrm{d})$, short-acting nifedipine and diazepam; according to the pharmacological group, employees for the nervous system. The factors that had a significant association $(p<0.05)$ with delirium were: living alone, institutionalized, hospitalized during the previous year, comorbidity, history of cerebrovascular disease, dementia, functional dependency, moderate to severe dehydration, and infection at admission to the emergency. It was obtained odds ratio > 1.0 for: high school degree or less education, history of cerebrovascular disease, liver cirrhosis, dementia and moderate to severe dehydration at income. Conclusions. They are risk factors for delirium (acute confusional state): high school degree or less education, history of cerebrovascular disease, liver cirrhosis, dementia and moderate to severe dehydration. The potentially inappropriate medication was not a risk factor for delirium in older adults.
\end{abstract}

KEY WORDS: inappropriate prescribing; delirium; acute confusional state; elderly 


\section{INTRODUCCIÓN}

En las últimas décadas, las mejoras económicas y en salud han producido disminución de la tasa de mortalidad e incremento de la expectativa de vida y, con ello, el aumento significativo de la población adulta mayor. En el Perú, los mayores de 65 años constituyen $6,4 \%$ de la población nacional, con tasa de crecimiento anual de 3,9\% (la más alta de los grupos etarios). En Lima y Callao se concentran $36 \%$ de esta población. ${ }^{1,2}$

Las múltiples enfermedades o factores de riesgo presentes en este grupo etario, obligan a emplear varios medicamentos durante periodos prolongados, por lo que se presentan problemas relacionados (sobreprescripción, desprescripción, subutilización, reacción adversa y adherencia), que incrementan la complejidad de estos pacientes. Se han definido listas explícitas de medicamentos (denominados medicamentos potencialmente inadecuados, criterios de Beers, STOPP, entre otros) con los cuales el riesgo de sufrir efectos adversos es superior al beneficio clínico, especialmente cuando existen alternativas terapéuticas más seguras $y / 0$ eficaces. ${ }^{2-4}$ Los criterios STOPP (Screening tool of older person's potentially inappropriate $)^{3,5}$ son prácticos y tienen mayor sensibilidad para identificar medicación potencialmente inadecuada (MPI).6,7

El síndrome confusional agudo (delírium) es frecuente en el adulto mayor. Se presenta entre $14 \%$ y $56 \%$, según estudios epidemiológicos, y en mayor frecuencia cuando se asocia con alguna enfermedad aguda de emergencia; produce mayor morbimortalidad, estancia hospitalaria, riesgo de institucionalización y deterioro funcional en los pacientes afectados. ${ }^{8-10}$

Según reportes internacionales, la frecuencia de prescripción inapropiada de fármacos en adultos mayores varía entre $14 \%$ y $37 \%$ en pacientes institucionalizados, con mayor frecuencia de efectos adversos, y es causa de $10 \%$ a $30 \%$ de los ingresos hospitalarios, con pérdidas económicas muy importantes, que produce un gran impacto en la salud pública, situación que está en incremento y que, en muchos casos, es inaceptable. ${ }^{11-17}$

El presente estudio tiene el objetivo de evaluar la asociación del delírium con la medicación potencialmente inadecuada.

\section{PACIENTES Y MÉTODO}

Estudio prospectivo analítico de casos y controles, realizado en pacientes de 65 años o más, que ingresaron por causas médicas al servicio de emergencia del Hospital Nacional Edgardo Rebagliati Martins, perteneciente al Seguro Social de Salud del Perú (EsSalud), en la ciudad de Lima.

Se considera caso al paciente con diagnóstico de delírium según los criterios del Confusion assessment method for the intensive care unit (CAM-ICU). ${ }^{18}$

Criterios de inclusión: 65 años o más, ingreso al servicio de emergencia por condiciones médicas, permanencia en el servicio de emergencia menor de 24 horas antes de la evaluación.

Criterios de exclusión: confusión mental por más de 72 horas antes del ingreso y provenir de algún servicio hospitalario en las 24 horas previas al ingreso. Criterios de eliminación: datos incompletos, fallecimiento en las primeras 48 horas de ingreso y alta voluntaria del servicio.

Mediante la fórmula para contraste de hipótesis, que compara dos proporciones $(Z$ y $Z$, con un nivel de significancia de 0,$05 ; \mathrm{p} 1=15 \%$ y p2 = 17\%), se calculó un tamaño muestral de 175 pacientes; los sujetos de estudio fueron seleccionados aleatoriamente, de manera secuencial, en el momento de su ingreso al servicio de emergencia, cada cinco días, durante los meses de enero a junio de 2011 (dos controles por caso). Se evaluaron 265 pacientes; de ellos, seis fueron excluidos por presentar confusión durante un lapso mayor de 72 horas y cinco por trasferencia de otro hospital; doce fueron eliminados del estudio por datos incompletos y cuatro por fallecimiento durante las primeras 48 horas.

Los pacientes seleccionados fueron sometidos a una evaluación inicial de su historia clínica de emergencia; a los que cumplieron los criterios de inclusión, se les realizó un examen clínico, entrevista con el cuidador (si el paciente no brindaba datos confiables), revisión de la historia clínica antigua y aplicación de los instrumentos de investigación. Los datos fueron recolectados por cuatro médicos internistas (entrenados) del servicio de emergencia. Finalmente, se consignó el destino y la estancia hospitalaria. 
El instrumento aplicado fue el CAM-ICU, ${ }^{18}$ usado para definir si cada paciente debía ser considerado un «caso» de delírium. En una prueba piloto, en 40 pacientes (que luego fueron incluidos en el estudio), se obtuvo adecuada validez del constructo, según el análisis factorial (medida de adecuación muestral de Kaiser-Meyer-Olkin $=0,948$, con nivel de significancia $p<0,001$ ) y según la matriz de componentes con método de extracción, que evalúa componentes principales: un solo componente que explique el $80 \%$ de la varianza. También se obtuvo una muy buena confiabilidad (coeficiente alfa de Cronbach $=0,917$ ). La ficha de recolección de datos se sometió al juicio de expertos.

Para la «medicación potencialmente inadecuada» se consideraron los criterios STOPP (Screening tool of older person's potentially inappropriate), 3,5 se preguntó al paciente o cuidador sobre la medicación habitual consumida durante los 15 días o más previos a su ingreso al servicio de emergencia; la información se complementó con lo registrado en la historia clínica antigua. Para evaluar el deterioro cognitivo se usó la escala SPMSQ(Shortportablemental status questionnarie) de Pfeiffer, ${ }^{19}$ aplicada en las primeras 24 horas del ingreso. Para la dependencia funcional se empleó el índice de Katz, ${ }^{20}$ que evalúa la capacidad para ejecutar actividades básicas de la vida diaria que el paciente tuvo durante la semana previa al ingreso. Para tener una evaluación geriátrica integral, también se calculó el índice de comorbilidad de Charlson, ${ }^{21}$ que permite pronosticar el riesgo de mortalidad en los siguientes diez años debido a las condiciones comórbidas.

Se revisó el correcto llenado de datos, que fueron codificados y procesados con el programa estadístico SPSS versión 17. Se realizó análisis bivariado; se emplearon las pruebas ji cuadrado, para las variables discretas, y t de Student, para las variables continuas. Se consideró diferencia significativa si $p<0,05$. Se calculó el odds ratio (OR), con un intervalo de confianza del $95 \%$, para variables consideradas significativas. Para controlar el posible efecto de las variables de confusión, se realizó el análisis multivariado de regresión logística binaria (evalúa cada factor mientras se mantienen constantes los demás factores), incluyendo a las variables que con el análisis bivariado obtuvieron $p<0,20$. Progresivamente se eliminaron los factores no significativos y finalmente se calculó el odds ratio.

Se obtuvo aprobación de la Oficina de Capacitación, Investigación y Docencia de la Red Rebagliati (EsSalud). Se solicitó consentimiento informado al paciente o cuidador, según los criterios de buenas prácticas clínicas. Se notificaba al médico tratante cuando se identificaba un caso de medicación potencialmente inadecuada, sugiriendo el retiro o cambio de la medicación.

\section{RESULTADOS}

Fueron evaluados 238 pacientes, 102 casos y 136 controles; $47,7 \%$ fueron del sexo masculino (48\% de los casos y $46,3 \%$ de los controles; $p=0,80$ ); la edad promedio fue 78,36 años $( \pm 7,83)$, entre 65 y 101 años (en los casos: 79,97 $\pm 8,3$ años; en los controles: 77,1 \pm 7,25 años; $p<0,01$ ).

Con relación al tipo de delírium: hipoactivo, 40\%; hiperactivo, $10 \%$ y mixto, $50 \%$. Las principales causas desencadenantes identificadas: infecciosa, en $48,5 \%$; metabólica, en $29,7 \%$ y otras (vasculares o hematológicas), $21,8 \%$.

En la Tabla 1 se muestran las características epidemiológicas: $1,7 \%$ del total de pacientes evaluados (4 controles) tenían actividad laboral remunerada. El informante de los datos fue el cuidador en $89 \%$ de casos y el paciente en $92 \%$ de controles. Los factores que en el análisis bivariado no mostraron asociación significativa $(p<0,05)$ fueron: mes de ingreso, antecedente de cáncer avanzado, falla renal crónica, polifarmacia, diagnóstico de ingreso, hemodiálisis y transfusión sanguínea reciente.

Los diagnósticos de ingreso más frecuentes en ambos grupos fueron: hipertensión arterial, diabetes mellitus, falla renal crónica, infección urinaria y neumonía. $16 \%$ de los pacientes presentaron más de tres diagnósticos de ingreso. El índice de comorbilidad de Charlson en el grupo de los casos fue de 3,1, en promedio, y en el de los controles, $2,54(p=0,99)$.

Se prescribieron 731 fármacos en 238 pacientes, que correspondieron a 113 principios activos de medicamentos; en promedio, tres fármacos por 
Tabla 1. Características epidemiológicas de los adultos mayores internados en el servicio de emergencia del HNERM (EsSalud), según presencia (casos) o ausencia (controles) del delírium, en 2011.

\begin{tabular}{|c|c|c|c|c|c|}
\hline \multirow[t]{2}{*}{ Característica } & \multicolumn{2}{|c|}{ Casos } & \multicolumn{2}{|c|}{ Controles } & \multirow[t]{2}{*}{$\mathrm{p}$} \\
\hline & $\mathrm{n}$ & $\%$ & $n$ & $\%$ & \\
\hline \multicolumn{6}{|l|}{$\triangle$ Sexo } \\
\hline - Femenino & 53 & 52 & 73 & 53,7 & 0,793 \\
\hline - Masculino & 49 & 48 & 63 & 46,3 & \\
\hline \multicolumn{6}{|l|}{$\Delta$ Edad } \\
\hline - 65-84 años & 70 & 68,6 & 113 & 83,1 & 0,009 \\
\hline - 85 a más & 32 & 31,4 & 23 & 16,9 & \\
\hline \multicolumn{6}{|l|}{$\Delta$ Grado de instrucción } \\
\hline - Secundaria o menor & 91 & 89,2 & 94 & 69,1 & 0,000 \\
\hline - Superior & 11 & 11,8 & 42 & 30,9 & \\
\hline \multicolumn{6}{|l|}{$\Delta$ Estado civil } \\
\hline - Soltero/viudo/divorciado & 52 & 51,0 & 45 & 33,1 & 0,005 (vive solo) \\
\hline - Casado & 50 & 49,0 & 91 & 66,9 & \\
\hline
\end{tabular}

paciente (3,27 en los casos [rango: 1 - 7] y 3,13 en los controles [rango: $0-10] ; p=0,324$ ). Los medicamentos usados con más frecuencia fueron: enalapril, ácido acetilsalicílico y furosemida; según subgrupo clínico: inhibidores de enzima convertidora de angiotensina, diuréticos "de techo alto", salicilatos y calcioantagonistas.

Se detectó medicación potencialmente inadecuada en 58 pacientes $(24,6 \%)$, cuya frecuencia según

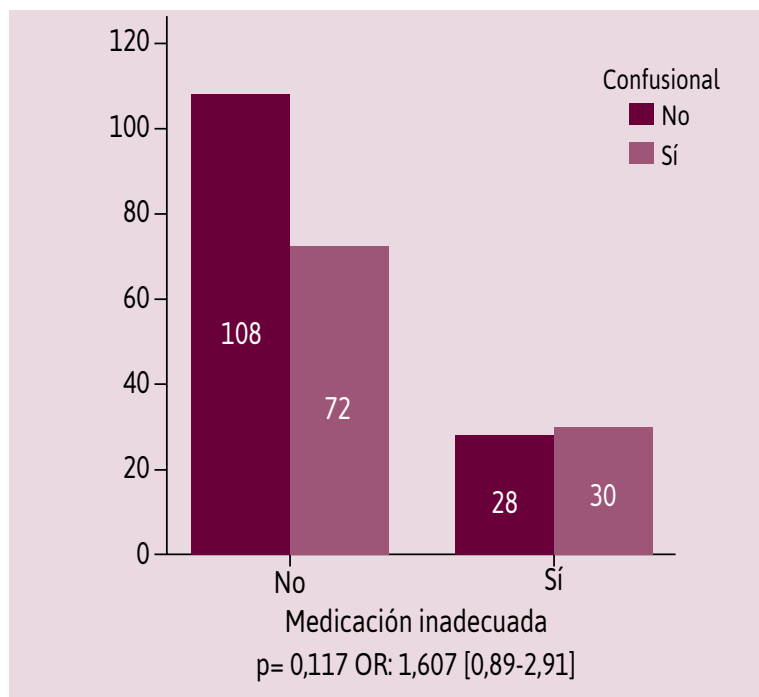

Figura 1. Medicación potencialmente inadecuada, según presencia 0 ausencia del delírium en adultos mayores internados en el servicio de emergencia del HNERM (EsSalud), en 2011. grupo de estudio se muestra en la Figura 1. En la Tabla 2 se presenta la relación de medicamentos potencialmente inadecuados más frecuentes. Según la clasificación anatómica, terapéutica y química de medicamentos, los más frecuentes fueron los utilizados para el sistema nervioso (21 pacientes), tracto alimentario y metabolismo (19) y sistema cardiovascular (11). Los resultados del análisis bivariado y multivariado con modelo de regresión logística se muestran en las Tablas 3 y 4.

\begin{tabular}{|c|c|c|c|c|}
\hline \multirow[t]{2}{*}{ Medicamento } & \multicolumn{2}{|c|}{$\begin{array}{l}\text { Casos } \\
(112)\end{array}$} & \multicolumn{2}{|c|}{$\begin{array}{c}\text { Controles } \\
(136)\end{array}$} \\
\hline & $\mathrm{n}$ & $\%$ & $n$ & $\%$ \\
\hline$\triangle$ Glibenclamida & 5 & 4,5 & 7 & 5,1 \\
\hline$\triangle$ Digoxina & 6 & 5,3 & 5 & 3,8 \\
\hline$\triangle$ Nifedipino $10 \mathrm{mg}$ & 3 & 2,7 & 5 & 3,8 \\
\hline$\triangle$ Diazepam & 3 & 2,7 & 2 & 1,5 \\
\hline$\triangle$ Risperidona & 3 & 2,7 & 1 & 0,7 \\
\hline$\Delta$ Terazocina & 2 & 1,8 & 2 & 1,5 \\
\hline$\triangle$ Amitriptilina & 2 & 1,8 & 1 & 0,7 \\
\hline$\triangle$ Biperideno & 2 & 1,8 & 1 & 0,7 \\
\hline$\triangle$ Nimodipino & 3 & 2,7 & 0 & 0 \\
\hline$\triangle$ Ibuprofeno & 0 & 0 & 2 & 1,5 \\
\hline
\end{tabular}


Tabla 3. Análisis de los factores asociados con el delírium en adultos mayores internados en el servicio de emergencia del HNERM (EsSalud), en 2011.

\begin{tabular}{|c|c|c|c|c|}
\hline \multirow[t]{2}{*}{ Factores } & \multicolumn{2}{|c|}{ Análisis bivariado } & \multicolumn{2}{|c|}{ Análisis de regresión* } \\
\hline & $\mathrm{OR}^{\natural}$ & IC $95 \%$ & OR & IC $95 \%$ \\
\hline$\triangle$ Edad $>85$ años & 2,246 & {$[1,217-4,147]$} & 0,492 & \\
\hline A Instrucción secundaria o menor & 2,37 & {$[1,373-4,092]$} & 3,054 & {$[1,282-7,274]$} \\
\hline $\begin{array}{l}\text { Estado civil: soltero/casado/ } \\
\text { divorciado (vive solo) }\end{array}$ & 1,39 & {$[1,087-1,780]$} & 0,715 & \\
\hline$\Delta$ Institucionalizado & 3,20 & {$[1,146-8,911]$} & 0,744 & \\
\hline$\Delta$ Internamiento previo & 1,28 & {$[1,036-1,590]$} & 0,872 & \\
\hline$\triangle$ Pluripatología & 1,43 & {$[1,140-1,781]$} & 0,710 & \\
\hline Cáncer avanzado & 1,36 & {$[0,897-2,073]$} & 1,597 & {$[0,670-3,807]$} \\
\hline$\triangle \mathrm{ECV}^{\#}$ previo & 4,96 & {$[2,501-9,837]$} & 3,729 & {$[1,475-9,428]$} \\
\hline$\Delta$ Cirrosis hepática & 1,92 & {$[0,866-4,267]$} & 4,428 & {$[1,523-12,875]$} \\
\hline$\triangle$ Demencia & 20,03 & {$[8,096-49,561]$} & 14,740 & {$[4,997-43,477]$} \\
\hline$\Delta$ Dependencia funcional & 2,99 & {$[1,745-5,112]$} & 1,253 & {$[0,555-2,832]$} \\
\hline$\Delta$ Deshidratación & 2,91 & {$[1,400-6,054]$} & 3,231 & {$[1,217-8,581]$} \\
\hline Infección al ingreso & 1,85 & {$[1,098-3,102]$} & 0,732 & \\
\hline$\triangle M P I^{s}$ & 1,607 & {$[0,886-2,914]$} & 0,847 & \\
\hline
\end{tabular}

* Análisis multivariado con regresión logística binaria.

\& OR: odds ratio. IC: intervalo de confianza.

\# ECV: enfermedad cerebrovascular.

\$ MPI: medicación potencialmente inadecuada.

\section{DISCUSIÓN}

Al analizar la relación entre el uso de medicación potencialmente inadecuada y el delírium (síndrome confusional agudo), se encontró mayor frecuencia de esta práctica en el grupo con confusión, pero la diferencia con el grupo control no fue significativa. Según los criterios STOPP, la medicación potencialmente inadecuada no alcanza significancia estadística en el presente trabajo para ser considerada un factor de riesgo para el delírium en la población estudiada. Pero, al considerar los

Tabla 4. Factores de riesgo para delírium en adultos mayores internados en el servicio de emergencia del HNERM (EsSalud), en 2011.

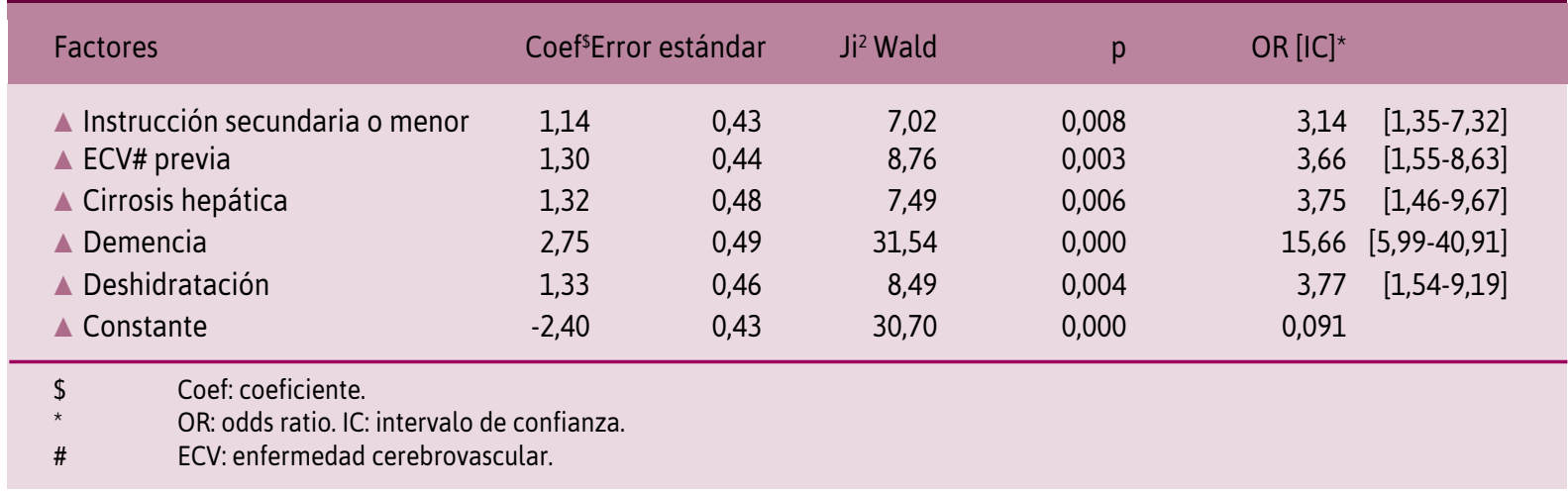


grupos de fármacos potencialmente inadecuados, vemos que el delírium se presentó con mayor frecuencia con el uso de fármacos para el sistema nervioso, similar a lo que encontraron Pisani et al.,22 en un estudio realizado con la Universidad de Yale, quienes consideran como factor de riesgo el uso de benzodiazepinas y opioides en pacientes de $\mathrm{UCl}$.

En lo referente a la medicación utilizada, el ácido acetilsalicílico es uno de los más frecuentes, similar a lo encontrado en otros estudios; esto se podría explicar por la alta frecuencia de enfermedad cerebrovascular como antecedente. Pero el uso de furosemida podría ser un factor contribuyente para la presentación del síndrome confusional, debido a la alteración metabólica que puede producir su consumo crónico. La diferencia con otros reportes es la baja frecuencia de analgésicos, probablemente por omisión y subrregistro; también, la baja frecuencia del uso de insulina y warfarina, por temor del paciente 0 del médico prescriptor. ${ }^{15}$ El uso de omeprazol es más frecuente en los pacientes que no presentaron síndrome confusional. No se obtuvo información sobre el tiempo de uso de este medicamento.

La tasa de medicación potencialmente inadecuada (24,6\%) fue ligeramente menor a lo reportado en otro de los hospitales nacionales más grandes en Lima, Perú, en 2005 (29,4\%) y 2011 (45,8\%), con mayor porcentaje en el grupo con síndrome confusional.,11 Un estudio realizado en Bogotá, Colombia, encontró una tasa más baja de MPI (21,5\%), pero fue en adultos mayores ambulatorios. ${ }^{12}$

Se encontró que entre los medicamentos potencialmente inadecuados más usados estuvieron, según el principio activo: glibenclamida, digoxina (en dosis mayores de $125 \mu \mathrm{g} / \mathrm{d}$ ), nifedipino de acción corta, benzodiacepinas de acción prolongada (diazepam), antipsicóticos (risperidona, amitriptilina) y terazocina. Los fármacos identificados como potencialmente inadecuados fueron similares a lo reportado por Oscanoa, ${ }^{4}$ a excepción de piroxicam, que actualmente no se encuentra en el petitorio de EsSalud. Pero en nuestro estudio aparece la glibenclamida como el más frecuente; pero recién en los últimos años fue clasificada como MPI.

Existe una alta frecuencia de uso de antidiabéticos orales, similar a lo reportado en América del Norte. ${ }^{14}$ Difiere del estudio de Colombia por la baja frecuencia de relajantes musculares, antihistamínicos anticolinérgicos y la asociación de anticoagulantes con aspirina. ${ }^{15}$

En América del Norte se reporta mayor riesgo de delírium o alucinaciones en los pacientes que recibieron narcóticos, pero no en los que recibieron anticolinérgicos; sin embargo, en este trabajo no se encuentra tal relación posiblemente por la baja frecuencia del uso de estos fármacos. ${ }^{14}$

Según el análisis bivariado, la cirrosis hepática avanzada no presentó influencia significativa; no obstante, su nivel de significancia aumenta en el análisis multivariado y se constituye en un factor de riesgo para desarrollar delírium, relación no mencionada en otros trabajos.

El síndrome confusional es ligeramente más frecuente en el sexo femenino, pero no se encontró diferencia significativa, semejante a otros estudios. ${ }^{9,23-26}$

La distribución etaria es similar al de otros reportes. Se observó que la edad es un factor asociado con el delírium; lo mismo reportan casi todos los otros estudios, excepto el realizado en los Países Bajos (probablemente porque se estudió a pacientes críticos y también fueron incluidos menores de 65 años). $9,10,24,26-28$

A diferencia de otros estudios, se encontró predominancia de la forma mixta del estado confusional agudo (50\%); en Barcelona, predominó la forma hiperactiva (68\%) y en Chile, la hipoactiva (72\%).24,27

Con relación a los factores de riesgo no farmacológicos, se halló que un alto nivel de educación se constituye en un efecto protector contra el delírium. El riesgo de delírium se eleva de manera significativa en pacientes que viven solos (solteros/casados/divorciados), que permanecen internados (institucionalizados), con antecedentes de varias hospitalizaciones previas, diagnóstico de enfermedad cerebrovascular y demencia, alto grado de dependencia funcional, diagnósticos de ingreso de infecciones (infección urinaria, neumonía), deshidratación moderada a severa y pluripatología. $1,6,10,11,24,26,27,29$

El término más difundido en nuestro medio para referirse a la medicación múltiple o con varios fármacos simultáneamente es "polifarmacia», 
pero encontramos que este factor no tiene relación con el desarrollo del síndrome confusional en el grupo estudiado, además de que es un término poco específico. Se identificó un amplio número de medicamentos utilizados por los pacientes, con un promedio de tres fármacos por cada uno, pero este es menor al de los otros estudios, probablemente por subregistro. ${ }^{4,6}$

Entre las limitaciones del presente estudio tenemos: los pacientes estudiados eran beneficiarios de un sistema de salud público, no se consideró en la medicación habitual el tiempo de tratamiento ni la adherencia al tratamiento y hubo un importante subrregistro de delírium y la medicación habitual en la historia clínica hospitalaria (antigua y de ingreso).

\section{CONCLUSIONES}

Se detectó medicación potencialmente inadecuada en $24,6 \%$ de los pacientes adultos mayores que ingresaron por causas médicas al servicio de emergencia del Hospital Nacional Edgardo Rebagliati Martins (EsSalud).

La medicación potencialmente inadecuada, según los criterios STOPP, no constituye un factor de riesgo para la presentación del delírium (síndrome confusional agudo) en adultos mayores.

Los factores de riesgo independientes para desarrollar delírium en adultos mayores internados en el servicio de emergencia son: instrucción secundaria o menor, enfermedad cerebro vascular previa, cirrosis hepática, demencia y deshidratación moderada a grave.

\section{REFERENCIAS BIBLIOGRÁFICAS}

I. Varela L, Chávez H, Herrera A, Méndez F, Gálvez M. Perfil del adulto mayor de Perú: desarrollando respuestas integradas de los sistemas de cuidados de la salud para una población en rápido envejecimiento. Intra II- Perú. Lima, Perú: OPS/OMS; 2004.

2. Oscanoa T, Castañeda B. Calidad de la prescripción farmacológica en geriatría: instrumentos de evaluación. $2^{\mathrm{a}}$ edición. Lima, Perú: Concytec; 2012.

3. Delgado E, Muñoz M, Montero B, Sánchez C, Gallagher PF y Cruz-Jentoft AJ. Prescripción inapropiada de medicamentos en los pacientes mayores: los criterios STOPP/START. Rev Esp Geriatr Gerontol. 2009;44(5):273-9.

4. Oscanoa T. Diagnóstico de problemas relacionados con medicamentos en adultos mayores al momento de ser hospitalizados. Rev Peru Med Exp Salud Publica. 20I I;28(2):25663.

5. Gallagher P, Ryan C, Byrne S, Kennedy J, O'Mahony D. STOPP (Screening Tool of Older Person's Prescriptions) and START (Screening Tool to Alert doctors to Right Treatment). Consensus validation. Int J Clin Pharmacol Ther. 2008;46(2):72-83.

6. Ryan C, O'Mahony D, Kennedy J, Weedle P, Byrne S. Potentially inappropriate prescribing in an Irish elderly population in primary care. Br J Clin Pharmacol. 2009;68(6):936-47.

7. Pattanaworasate W, Emmerton L, Pulver L, Winckel K. Comparison of prescribing criteria in hospitalised Australian elderly. Pharm Pract (Granada). 2010;8(2): 132-8.

8. Inouye SK. Delirium in older persons. N Engl J Med. 2006;354(II):II57-65.

9. Ortiz P, Chávez JC, Chávez H, Varela LF. Delirio en el paciente adulto mayor hospitalizado: presentación clínica y morbimortalidad. Rev Soc Peru Med Inter. 2002; 14(4): 184-90.

10. Valera L, Chávez H, Gálvez M, Méndez F. Características del deterioro cognitivo en el adulto mayor hospitalizado a nivel nacional. Rev Soc Peru Med Inter. 2004; 17(2):37-42.

II. Oscanoa T]. Prescripción potencialmente inadecuada de medicamentos en adultos mayores [tesis doctoral]. Lima, Perú: Unidad de Posgrado de la Universidad Nacional Mayor de San Marcos; 2005.

12. Holguín-Hernández E, Orozco-Díaz G. Medicación potencialmente inapropiada en ancianos de un hospital de primer nivel, Bogotá 2007. Rev Salud Pública. 2010;12(2):287-99.

13. Fiss T, Dreier A, Meinke C, van den Berg N, Ritter CA, Hoffmann $W$. Frequency of inappropriate drugs in primary care: analysis of a sample of immobile patients who received periodic home visits. Age Ageing. 20I I;40(I):66-73.

14. Stockl KM, Le L, Zhang S, Harada AS. Clinical and economic outcomes associated with potentially inappropriate prescribing in the elderly.Am J Manag Care. 2010;16(I):el-10.

15. Budnitz DS, Lovegrove MC, Shehab N, Richards CL. Emergency hospitalizations for adverse drug events in older Americans. N Engl J Med. 20I I;365(2I):2002-I2.

16. Wu TY, Jen MH, Bottle A, Molokhia M, Aylin P, Bell D, et al. Tenyear trends in hospital admissions for adverse drug reactions in England 1999-2009.J R Soc Med. 2010;103(6):239-50.

17. Hamilton HJ, Gallagher PF,O'Mahony D. Inappropriate prescribing and adverse drug events in older people. BMC Geriatr. 2009;9:5.

18. Inouye SK, van Dyck CH, Alessi CA, Balkin S, Siegal AP, Horwitz RI. Clarifying confusion: the confusion assessment method. A new method for detection of delirium. Ann Intern Med. 1990 Dec 15; I I3(I2):94I-8.

19. Pfeiffer E. A short portable mental status questionnaire for the assessment of organic brain deficit in elderly patients. J Am Geriatr Soc. 1975;23(10):433-4I.

20. Katz S, Ford AB, Moskowitz RW, Jackson BA, Jaffe MW. Studies of illness in the aged. The index of $A D L$ : a standardized measure of biological and psychosocial function. JAMA. 1963;185:9|4-9.

21. Charlson ME, Pompei P,Ales KL, MacKenzie CR. A new method of classifying prognostic comorbidity in longitudinal studies: development and validation. J Chronic Dis. 1987;40(5):373-83.

22. Pisani MA, Murphy TE, Araujo KL, Slattum P,Van Ness PH, Inouye SK. Benzodiazepine and opioid use and the duration of intensive care unit delirium in an older population. Crit Care Med. 2009;37(I): 177-83.

23. Amado JP, Chucas LA, Rojas CR, Pintado S, Cerrón CA, Vásquez R. Factores asociados a síndrome confusional agudo en adultos mayores internados en emergencia de un hospital terciario. An Fac Med. 2013;74(3): 193-7.

24. Formiga F, Marcos E, Sole A, Valencia E, Lora-Tamayo J, Pujol R. Síndrome confusional agudo en pacientes ancianos ingresados por patología médica. Rev Clin Esp. 2005;205(I0):484-8.

25. Laurila JV, Pitkala KH, Strandberg TE, Tilvis RS. Detection and documentation of dementia and delirium in acute geriatric wards. Gen Hosp Psychiatry. 2004;26(I):31-5. 
26. Lama J, Valera L, Ortiz PJ. Prevalencia y factores de riesgo del estado confusional agudo en el adulto mayor en una sala de emergencias médicas. Rev Med Hered. 2002;13(I):10-8.

27. Carrasco M, Hoyl T, Marín PP, Hidalgo J, Lagos C, Longton C, et al. Subdiagnóstico de delirium en adultos mayores hospitalizados. Rev Med Chil. 2005; 133(I2):1449-54.

28. Cole MG, McCusker J, Bellavance F, Primeau FJ, Bailey RF, Bonnycastle MJ, et al. Systematic detection and multidisciplinary care of delirium in older medical in patients: a randomized trial. CMAJ. 2002;167(7):753-9.

29. Grover S, Subodh BN, Avasthi A, Chakrabarti S, Kumar S, Sharan $\mathrm{P}$, et al. Prevalence and clinical profile of delirium: a study from a tertiary-care hospital in north India. Gen Hosp Psychiatry. 2009;3I(I):25-9.

\section{Correspondencia \\ Dr. José Percy Amado Tineo \\ jpamadot@hotmail.com}

Conflictos de interés

Los autores declaran no tener conflictos de interés durante el planteamiento, ejecución de la investigación y la elaboración del artículo para su publicación.

Fecha de recepción: 2 de agosto de 2015

Fecha de aceptación: 2 de noviembre de 2015 